ARTÍCULO

\title{
Evaluación de un programa de apoyo psico-social en torno a los conceptos de persistencia y retención universitaria
}

\author{
Pablo Castillo Armijo' (D) \\ Tomas Morales Zárate" (D) \\ Carlos Miranda Carvajal'I (D)
}

\section{RESUMEN}

El alto número de estudiantes que fracasa el primer año en educación superior en Chile ha llevado al Ministerio de Educación a crear estrategias de acompañamiento, como forma de elevar índices de retención y evitar la deserción universitaria. Se buscó comprender los significados y alcances del fenómeno de persistencia y retención universitaria, utilizando una metodología cualitativa de estudio de casos (Stake, 1998) desde una perspectiva comunicativo-crítica (Gómez et al., 2006). Los participantes fueron los estudiantes de primer año (cohorte 2015) pertenecientes al programa "me quedo en la universidad". Se constatan diversas evaluaciones positivas y negativas del programa, destacando aspectos de participación, estrategias de aprendizaje, factores motivacionales e incluso elementos como el espacio y la identidad que puedan llegar a establecer con la universidad o con su carrera, que pueden influir en la decisión de mantenerse o desertar del sistema.

PALABRAS CLAVE

retención estudiantil; deserción estudiantil; educación superior; acompañamiento; universidad pública.

'Universidad de Talca, Talca, Chile.

"Universidad de Valparaíso, Valparaíso, Chile.

"'Universidad de Playa Ancha, Playa Ancha, Chile. 


\title{
EVALUATION OF A PSYCHO-SOCIAL SUPPORT PROGRAM ABOUT CONCEPTS OF PERSISTENCE AND UNIVERSITY RETENTION
}

\begin{abstract}
The high number of students who fail their first year of higher education in Chile has led the Ministry of Education to create strategies of accompaniment, as a way to raise retention rates and prevent university dropouts. We sought to understand the meanings and scope of the persistence and university retention phenomenon, using a qualitative case study methodology (Stake, 1998) from a communicative-critical perspective (Gómez et al., 2006). Participants were freshmen (cohort 2015) belonging to the program "me quedo en la universidad" ("I stay in University"). Various positive and negative evaluations of the program are noted, highlighting aspects of participation, learning strategies, motivational factors and even elements such as space and identity that may come to be established with the university or with their career, which may influence the decision to continue or defect from the system.
\end{abstract}

\section{KEYWORDS}

student retention; student dropout; higher education; accompaniment; public university.

\section{AVALIAÇÃO DE UM PROGRAMA DE APOIO PSICOSSOCIAL EM RELAÇÃO AOS CONCEITOS DE PERSISTÊNCIA E RETENÇÃO UNIVERSITÁRIA}

\section{RESUMO}

$\mathrm{O}$ alto número de alunos que fracassam no primeiro ano do ensino superior no Chile levou o Ministério da Educação a criar estratégias de acompanhamento, como forma de elevar os índices de retenção e evitar a evasão em universidades. Procurou-se compreender os significados e alcances do fenômeno da persistência e da retenção universitária, utilizando uma metodologia qualitativa de estudo de caso (Stake, 1998) a partir de uma perspectiva crítico-comunicativa (Gómez et al., 2006). Os participantes foram os alunos do primeiro ano (cohorte 2015) pertencentes ao programa "me quedo en la universidad" ("Eu fico na universidade"). Várias avaliações positivas e negativas do programa são constatadas, destacando aspectos de participação, estratégias de aprendizagem, fatores motivacionais, e até mesmo elementos como espaço e identidade que podem ser estabelecidos com a universidade ou seu curso, que podem influenciar a decisão de ficar ou abandonar o sistema.

\section{PALAVRAS-CHAVE}

retenção estudantil; evasão estudantil; ensino superior; acompanhamento; universidade pública. 


\section{INTRODUCCIÓN Y JUSTIFICACIÓN DE LA INVESTIGACIÓN}

La reforma chilena de Educación Superior de 1980, establece una estructura piramidal de funcionamiento de las Instituciones de Educación Superior, donde los Centros de Formación Técnica (CFT) estarían en la base, luego los Institutos Profesionales (IP) en medio y en la cúspide las Universidades sean estas Estatales y Privadas con o sin fines de lucro. El Estado chileno de esta forma, comienza un proceso de entrega gradual de recursos a entes privados que generó la masificación del sistema de educación superior chileno y la gran expansión de la matrícula, especialmente en las universidades en los últimos años (Campbell Esquivel, 1995).

Esta gran reforma de corte neoliberal originada durante la dictadura, implicó por una parte que más estudiantes vulnerables social y económicamente pudiesen acceder a la educación superior, que parece ser todo un éxito en masificación y acceso, pero que a la larga ha generado endeudamiento de esas mismas familias y una alta tasa de fracaso y deserción de esos mismos estudiantes que la OCDE (2009) cifró en cerca del $50 \%$ para los dos primeros quintiles, es decir, los más pobres.

El Ministerio de Educación del Gobierno de Chile (MINEDUC), a través del Servicio de Información de Educación Superior (SIES, 2013), ha calculado que un $36 \%$ de los estudiantes no continúa luego del primer año de estudios, lo que aumenta a un $43 \%$ al segundo año. El mismo estudio analizó la cohorte 2008 por un período de tres años y determinó que el 17,2\% es la deserción definitiva y existe un porcentaje considerable de reingresos. La mala noticia es que los que mayormente desertan son los estudiantes provenientes de colegios municipales, donde se concentra una gran población vulnerable.

El MINEDUC luego de estas cifras negativas ha declarado como prioridad establecer políticas educativas tendientes a mejorar la retención de estudiantes en la Educación Superior, en especial por el alto coste económico y social que esto tiene para los estudiantes, familias y que afecta a la propia eficiencia del Estado (SIES, 2013).

Es así, que la deserción universitaria se transforma en uno de los mayores desafíos a las políticas públicas en materia de reforma a la educación superior chilena, pero que no ha sido abordado como foco problemático por las instituciones de educación superior, salvo el caso de la Universidad de Santiago de Chile (Donoso, Donoso y Frites, 2013).

Hoy en día nos encontramos con voluntades políticas desde el poder Ejecutivo y de los partidos políticos por instalar una reforma estructural de la Educación Superior en Chile que aspira entre otras cosas a transformar su estructura, pero manteniendo su lógica de mercantilización afianzando valores de competencia y eficiencia (Orellana, Sanhueza y Gajardo, 2016). Esta instrumentalización de la educación agudiza la segregación cultural por medio del rentismo que establece el Estado - con recursos públicos - con los sectores privados del país (Riesco, 2010). Como consecuencia de lo anterior se prolonga aún más un sistema educacional excluyente, que ahonda en la segregación social y que sigue aspirando como un idealismo disfrazado a diseñar políticas públicas capaces de permitir que todos los jóvenes y adultos puedan acceder a la educación sin importar su capacidad de pago 
y endeudamiento, no previendo los apoyos necesarios para nivelar conocimientos y prevenir de esta forma la deserción (Donoso, Donoso y Frites, 2013).

Por otra parte, se puede decir que no existe un diseño político que reconozca las falencias estructurales de la educación y por tanto carente de soportes hacia los estudiantes y sus familias (Sánchez, 2015). Situación que genera un sistema de exclusión de miles de estudiantes que requiriendo apoyo en este tránsito de la educación media a la universitaria simplemente quedan desamparados a su suerte.

Los últimos años el MINEDUC (Chile, 2013b) ha comenzado a abordar el fenómeno de la deserción universitaria con acciones de apoyo y atención preferencial a los primeros años que ingresan a la universidad. Estos programas de apoyo psico-social tienen diversas características en la aplicación, algunos con focos en carreras específicas con alta deserción y otras destinadas al acompañamiento individual de estudiantes vulnerados socialmente, como es el caso del Programa de Acompañamiento y Acceso Efectivo a la Educación Superior (PACE) ${ }^{1}$.

El presente artículo refleja una realidad particular de una universidad pública chilena que ha implementado uno de estos programas de apoyo psico-social y que en voz y experiencia de los estudiantes que fueron atendidos tendría diversas consecuencias y ponderaciones.

\section{RETENCIÓN, PERSISTENCIA Y COBERTURA EN LA EDUCACIÓN SUPERIOR}

Los antecedentes contextuales acerca del fenómeno de retención y persistencia educacional se sitúan como estudios dominantes sobre los resultados educacionales impulsados hasta la década de los 70's a nivel mundial, siendo la deserción o el abandono el tema predominante a estudiar. En general, estos estudios se referían básicamente a los niveles primario y secundario, aunque la Educación Superior no estaba ajena al tema, en particular en Chile, donde ya existía una preocupación por la materia (Ahumada, 1958; Hamuy, 1961; Chile, 1964; Schiefelbein, 1976), lo que no implica que este problema en América Latina haya sido superado, sino por el contrario, como lo sintetiza Gajardo (2005) se trata de un fenómeno demasiado presente en la realidad educativa del continente.

Autores como Himmel (2002) y Barrios (2011) son los expositores de esta tendencia conceptual, y que definen la deserción como el "abandono prematuro de un programa de estudios antes de alcanzar el título o grado, y considera un tiempo suficientemente largo como para descartar la posibilidad de que el estudiante se reincorpore” (Himmel, 2002, p. 95). Siguiendo en esta línea, Stratton, O’Toole y Wetzel (2008), plantean el distanciamiento entre la deserción y la suspensión, explicando que en algunos casos los estudiantes hacen una pausa en sus estudios, tras lo que se reincorporan al sistema. Es posible entonces distinguir dentro de la deserción diversas categorías dependiendo de las circunstancias y motivaciones que la originaron.

1 El programa PACE garantiza cupos en la educación superior a los estudiantes de sectores vulnerables, que cumplan los requisitos de excelencia académica. 
Considerando dos elementos sustanciales con que el MINEDUC (Chile, 2013b) comprende este fenómeno se puede desglosar lo siguiente:

- entiende la deserción como el abandono (voluntario o no) prematuro de un programa de estudios antes de lograr la graduación;

- define la retención como la otra cara de la moneda de la deserción, definición dicotómica según Barrios (2011). Esta dicotomía plantea el autor, contrapone ambos elementos de este proceso diferenciándolos uno de otros.

Tanto Barrios (2011) y como Himmel (2002) resumen los principales enfoques que facilitan la comprensión del fenómeno a partir de sus características:

- enfoque psicológico: este enfoque intenta explicar la deserción a partir de las diferencias que pueden encontrarse entre los rasgos de personalidad de quienes completan sus estudios y de quienes los abandonan. Attinasi (1986) explica que la deserción también está determinada por la evaluación que hacen los estudiantes de su vida una vez ingresado a la Educación Superior. Ethington (1990) demostró que empíricamente que el rendimiento académico previo, afecta sustancialmente los resultados futuros de los estudiantes;

- enfoque económico: este modelo se centraliza en la idea de que los estudiantes permanecen en la Educación Superior en la medida en que los beneficios tanto sociales como económicos sean mayores que los costos derivados de ello. En general esta línea investigativa se basa en los estudios que examinan los programas de rebajas de matrículas, crédito y becas siendo posiblemente la línea de investigación más recurrida para entender este fenómeno (St. John et al., 2000);

- enfoque sociológico: centrada en los elementos que afectan externamente al individuo. El representante más reconocido es Spady (1970) y su modelo de integración social (Pascarella, 1985; Tinto, 1975) quien lo baso en la teoría de suicidio de Durkheim (2012). Según esta teoría, el suicidio se produce debido a la ruptura del individuo con el sistema social, porque no ha sido capaz de integrarse a la sociedad. Al respecto Spady (1970) propuso que la deserción es entendida como el resultado de la falta de integración de los alumnos a los sistemas de Educación Superior;

- enfoque organizacional: la lógica organizacional define que la deserción depende principalmente de las características de la Institución de Educación Superior. Como señalan Braxton, Milem y Sullivan (2000) los factores que influyen en el abandono de la educación superior son la calidad de los docentes y la experiencia de los estudiantes en la sala de clase. Otros académicos como Tillman (2002) añade los factores de seguro de salud, actividades deportivas y culturales, calidad de las bibliotecas, infraestructura e indicadores de la cantidad de estudiantes por docente;

- enfoque de integración: Vincent Tinto (1975) afirma que los estudiantes construyen su integración social y académica evitando conductas que les 
generan costos de algún tipo y buscando recompensas en las relaciones, interacciones y estados emocionales. Las variables socio-económicas y culturales de la familia, sus valores, sus atributos personales y la experiencia académica previa a la Educación Superior influyen sobre las probabilidades de deserción.

Para el caso chileno, el MINEDUC (Chile, 2013b) señala que el acceso a educación superior en los últimos 30 años experimentó un gran crecimiento a nivel de pregrado, un 600\% para el año 2012. No obstante para el mismo año, los datos reflejan que más de un 20\% de quienes ingresaron en la Educación Superior no concluyeron el programa en el que inicialmente se habían matriculado.

La investigación de Donoso y Schiefelbein (2007) establecen que la retención de estudiantes en la educación universitaria implica comprender un fenómeno más amplio y que se relaciona con políticas de acceso y selección en la educación superior tradicional. Los autores señalan que el sistema de selección de estudiantes responde a la hipótesis que no todos los egresados de la enseñanza media poseen las habilidades, condiciones, capacidades, aptitudes o competencias para proseguir sus estudios universitarios (Donoso y Schiefelbein, 2007).

Navarrete, Candia y Puchi (2013) afirman que la deserción afectó la comprensión de esta problemática sobre las capacidades de ingreso que tienen los estudiantes y redujo la atención sobre el tema de fondo que es la retención de estudiantes. Por consiguiente, los cambios orientados para potenciar la investigación de este fenómeno son difíciles de entablar y más aún cuando no hay claridad de lo que se tiene que investigar (Cuchacovich, 2011).

La matrícula en IES aumentó de 668.853 el año 2006 a 1.168.901 el año 2016, es decir un 74,76\% según datos del Consejo Nacional de Educación (CNED, 2018). Aunque se haya registrado un constante crecimiento en la cobertura de la educación superior en Chile en las últimas décadas, siguen existiendo importantes diferencias por nivel socioeconómico en el acceso a este nivel educativo. Según la Encuesta CASEN (Gobierno de Chile, 2011), la tasa neta de cobertura en educación superior en el quintil de mayores ingresos es del 59\%, mientras que en el primer quintil esta cifra es del 22\% (Chile, 2013a). Esta diferencia cobra mayor importancia cuando además se considera la matrícula de alumnos de distinto nivel socioeconómico por tipo de institución.

La Organización para la Cooperación y el Desarrollo Económicos (OCDE, 2009 , p. 83) destaca en un informe que en Chile "existen marcadas diferencias en el tipo de institución a la que acceden los jóvenes según su nivel socioeconómico, siendo los estudiantes de menores recursos mucho más proclives a matricularse en instituciones técnicas, las que en su mayoría no están acreditadas" (apud Servicio de Información de Educación Superior, 2013).

Un estudio de Castillo y Cabezas (2010), que caracterizó a los jóvenes de primera generación que ingresan a estudiar a la Educación Superior en Chile, es decir, con padres sin estudios terciarios, mostró resultados similares al evidenciar diferencias en el tipo de institución en la que se matriculan estos estudiantes y al compararlos con sus pares de padres que sí alcanzaron la educación terciaria. 
En el fondo, el crecimiento de la cobertura de acceso a la educación superior ha evidenciado que las cifras de abandono o deserción universitaria han aumentado considerablemente, aumentando las brechas de éxito y fracaso académico entre estudiantes con mayores y menores condiciones socio-culturales.

La altísima tasa de deserción es la mejor prueba del fracaso del modelo neoliberal aplicado a la educación superior. El aumento de cobertura es en realidad solo aparente pues una cantidad muy importante de los alumnos que ingresan no llegan a titularse - por sobre el $45 \%$ - y, lo que es más grave, terminan endeudados y, por cierto, frustrados. Adicionalmente, la deserción golpea con más fuerza a los alumnos de más bajos ingresos (Basso, 2016).

Aquello se corrobora con las cifras que entregó el Sistema de Información de Educación Superior (SIES, 2013) al analizar la permanencia de los alumnos en la misma institución y encuentra que, al segundo año un $38 \%$ de los estudiantes universitarios, un 55\% de los estudiantes de Instituto Profesionales (IP) y un 53\% de los estudiantes de Centros de Formación Técnica (CFT) ya no se encuentra matriculado en la misma IES a la que ingresaron. La explicación que da el SIES establece que este fracaso se produce por la falta de soporte académico, por la inexistencia de políticas reales de apoyo estudiantil, un sistema de beca muy restringido y, además, un elevado costo arancelario de las carreras.

En conclusión el fenómeno de la deserción no solo afecta al individuo sino que trae algunas consecuencias sociales como por ejemplo, afecta las expectativas de los estudiantes y sus familias; afecta emocionalmente por la disonancia entre las aspiraciones de los jóvenes y sus logros y trae consecuencias económicas tanto para la persona como para el sistema en conjunto (González, 2005).

\section{CONTEXTUALIZACIÓN DEL CASO “ME QUEDO EN LA UNIVERSIDAD: HACIA MAYOR EQUIDAD E INCLUSIÓN UNIVERSITARIA”}

En efecto, este estudio de caso focaliza el fenómeno de deserción en una universidad estatal chilena, de la primera cohorte de estudiantes que participaron en el programa de retención universitaria durante el transcurso del año académico 2015, pertenecientes a las carreras con menor índice de retención estudiantil de la universidad analizada y que responde a las directrices de un Programa de Becas de Nivelación Académica (BNA) entrega por el Gobierno de Chile.

Este programa titulado "Me quedo en la universidad: Hacia mayor equidad e inclusión universitaria" forma parte de un Proyecto de Mejoramiento Institucional (PMI) que apunta a profundizar la implementación de las estrategias institucionales de apoyo académico y psico-social. La universidad tuvo como principal objetivo fortalecer y profundizar la atención preferencial hacia los estudiantes de primer año, por tanto la propuesta de trabajo propuso dos áreas de nivelación; desde el ámbito cognitivo (fortalecimiento de estrategias de aprendizaje, desarrollo de hábitos de estudios, fortalecimiento de estrategias de autorregulación y competencias discursivas) y desde el ámbito socio-afectivo (favoreciendo proceso de adaptación al mundo universitario a partir del acompañamiento en los procesos afectivos que 
influyen en el aprendizaje y el rendimiento académico). Las principales actividades que implanto el programa fueron:

Levantamiento de perfiles y caracterización de estudiantes BNA (primer semestre); basándose en los instrumentos evaluativos de la universidad que permiten conocer a los estudiantes de primer año (encuesta de caracterización, test de habilidades discursivas y el test de autorregulación), que generaron base de datos para la planificación de las intervenciones y trabajo para el primer año. La BNA, permite profundizar en aspectos psicosociales y cognitivos que afinen la caracterización de los estudiantes pertenecientes al programa y trabajar más focalizadamente en torno a esas necesidades. Es por ello que se suman los instrumentos de resiliencia (autoestima de Coopersmith y estilos de aprendizaje de Schmeck) además de test vocacional, que se trabajaron en los respectivos talleres de inserción universitaria.

Bienvenida e inducción a la vida universitaria a partir de la metodología de talleres (primer mes); Se entrega la información correspondiente a beneficios, responsabilidades e implicaciones del programa para los estudiantes y sus familias, además se realizan talleres de inducción universitaria, cuyo fin es desarrollar estrategia de adaptación cognitiva, afectiva y social que implique comprender y significar las experiencias de iniciar una vida universitaria, desenvolverse e interactuar eficazmente al interior de la universidad y la comunidad en la que se insertó. Esta metodología de talleres busca el fortalecimiento del proyecto de vida universitaria tratando temas como: obstáculos y expectativas al iniciar esta nueva etapa de vida, regulación emocional, autoestima académica y modos de enfrentar los primeros resultados académicos, vinculación con la comunidad local, entre otros. Finalmente para retroalimentar y mejorar esta metodología de inducción se dispuso de evaluaciones de los talleres para sistematizar las experiencias a modo grupal y una encuesta de satisfacción.

Metodología de seminarios; realización de seminarios temáticos internos a autoridades, académicos, profesionales de apoyo y comunidad mentora BNA, que permita generar espacios de reflexión en torno a los procesos involucrados en la inserción universitaria. La metodología de trabajos utilizada es: expositivas, metodología activa participativa y metodología reflexiva-propositiva. Esta última metodología insta al estudiante a que produzca un conocimiento propositivo y transformador por medio del debate de ideas, reflexión y construcción colectiva de conocimiento entre los distintos actores de la universidad.

Estrategia de formación de comunidades de aprendizaje y guía de estudiantes mentores; deben formar comunidades de aprendizajes heterogéneas en su proceso de inserción a la vida universitaria, este proceso será mediado por estudiantes de años superiores (mentores) que cuentan con competencias y habilidades que les permiten transmitir conocimientos disciplinares específicos y experiencias relevantes. Sus funciones son acompañar y guiar a los estudiantes del "Me quedo en la universidad" Algunos de los temas establecidos en las mentorías fueron: organización del tiempo de estudio, hábitos de estudio, estilos y estrategias cognitivas para el aprendizaje, apoyar académico asignaturas críticas.

Seguimiento del proceso de adaptación a la vida universitaria; a partir de la confección de reportes psicosociales y académicos del proceso de inserción de 
los estudiantes becarios del "Me quedo en la universidad", realizado por profesionales del área de la psicología con el objetivo de indagar en los factores que están influyendo negativa y/o positivamente en la adaptación. Los ámbitos a indagar en los becarios fueron: hábitos de estudios, estrategias de aprendizajes, problemáticas familiares, orientación vocacional, dificultades académicas, regulación emocional, entre otras.

Atención de orientación vocacional; se realizan atenciones individuales, en pequeños grupos o talleres masivos con los orientadores para abordar alguna temática psico-educativa que dificulte su desempeño, o en caso de que se detecte algún trastorno de aprendizaje específico también se dispone de un profesional especialista en psicopedagogía con experiencia en jóvenes y adultos.

Tutorías académicas; se focalizan en aquellos becarios que requieran de un apoyo académico personalizado en asignaturas de nivelación de competencias básicas y que no se encuentren cubiertas por otras instancias de apoyo institucional, estas se llevarán a cabo una vez generada la alerta temprana. Esta estrategia pedagógica focalizada consiste en una tutoría profesional del área con un máximo de cuatro becarios a su cargo.

\section{METODOLOGÍA DE LA INVESTIGACIÓN}

\section{OBJETIVOS DE LA INVESTIGACIÓN}

\section{Objetivo general}

Comprender los significados y alcances del fenómeno de persistencia y retención universitaria a partir de la experiencia de los estudiantes de la cohorte 2015 que participaron del programa "Me quedo en la universidad".

\section{Objetivos específicos}

- Evaluar la implementación del programa de apoyo psico-social por parte de los estudiantes;

- Analizar las distintas perspectivas que asumen los estudiantes frente al proceso de retención y su contexto universitario.

\section{ENFOQUE DE INVESTIGACIÓN}

Según los objetivos de investigación, la opción óptima para aproximar a las interpretaciones y descripciones del fenómeno social es utilizar un enfoque metodológico cualitativo que se caracteriza por comprender la práctica social, por medio del ejercicio de relacionar y ordenar lógicamente la información que la misma practica social suministra en el escenario. El enfoque cualitativo se fundamenta en la sistematización de la importancia de encontrar el significado y la comprensión de la práctica social, a través de ordenar y relacionar lógicamente la información que la práctica suministra y que se registra. Esta investigación de corte cualitativo, corresponde a un estudio de casos (Stake, 1998) con un enfoque comunicativo-crítico (Gómez et al., 2006). 


\section{PARTICIPANTES E INSTRUMENTOS DE RECOLECCIÓN DE INFORMACIÓN}

Considerando la validez de los estudios actuales de retención estudiantil, donde el margen analítico comprende de primer año hasta tercer año, es que se optó por escoger a estudiantes matriculados el año 2015 que cursaron su primer año de universidad, y que para el año 2016 han permanecido en la institución. Así que básicamente el centro de este estudio se focalizó en la cohorte 2015, porque en él se materializan los fenómenos y las experiencias más claras sobre la efectividad de este plan de retención.

En este caso se recolectó una base de datos de 12 voluntarios de las tres carreras con mayor índice de deserción (Trabajo Social, Diseño e Ingeniería Civil Biomédica). Durante el proceso de recolección de voluntarios, 9 aceptaron la convocatoria y quienes finalmente se convirtieron en el muestreo del estudio, siendo 4 de la carrera de Trabajo Social, 3 de la carrera de Diseño y 2 de la carrera de Ingeniería Civil Biomédica (Cuadro 1).

Cuadro 1 - Esquema de técnicas, códigos asignados y perfil de los participantes.

\begin{tabular}{|c|c|c|}
\hline \multirow{10}{*}{$\begin{array}{l}\text { Entrevista } \\
\text { individual }\end{array}$} & Código asignado & Perfil Participante \\
\hline & Estudiante 1 & $\begin{array}{l}\text { Estudiante de la carrera de Diseño. Proveniente de la comuna } \\
\text { de Santa Cruz, VI Región, Chile. Estudió en un Colegio } \\
\text { Particular Subvencionado. }\end{array}$ \\
\hline & Estudiante 2 & $\begin{array}{c}\text { Estudiante de la carrera de Trabajo Social. Proveniente de la } \\
\text { comuna de Osorno, X Región, Chile. Estudió en un Colegio } \\
\text { Municipal. }\end{array}$ \\
\hline & Estudiante 3 & $\begin{array}{c}\text { Estudiante de la carrera de Ingeniería Civil Biomédica. } \\
\text { Proveniente de la comuna de Valparaíso, V Región, Chile. } \\
\text { Estudió en un Particular Subvencionado. }\end{array}$ \\
\hline & Estudiante 4 & $\begin{array}{c}\text { Estudiante de la carrera de Diseño. Proveniente de la comuna } \\
\text { de Viña del Mar, V Región, Chile. Estudió en un Colegio } \\
\text { Particular Subvencionado. }\end{array}$ \\
\hline & Estudiante 5 & $\begin{array}{c}\text { Estudiante de la carrera de Diseño. Proveniente de la comuna } \\
\text { de Viña del Mar, V Región, Chile. Estudió en un Colegio } \\
\text { Particular Subvencionado. }\end{array}$ \\
\hline & Estudiante 6 & $\begin{array}{c}\text { Estudiante de la carrera de Trabajo Social. Proveniente de } \\
\text { la comuna de Valparaíso, V Región, Chile. Estudió en un } \\
\text { Colegio Municipal. }\end{array}$ \\
\hline & Estudiante 7 & $\begin{array}{l}\text { Estudiante de la carrera de Ingeniería Civil Biomédica. } \\
\text { Proveniente de la comuna de Quillota, V Región, Chile. } \\
\text { Estudió en un Colegio Particular Subvencionado. }\end{array}$ \\
\hline & Estudiante 8 & $\begin{array}{c}\text { Estudiante de la carrera de Trabajo Social. Proveniente de la } \\
\text { comuna de Cabildo, V Región, Chile. Estudió en un Colegio } \\
\text { Municipal. }\end{array}$ \\
\hline & Estudiante 9 & $\begin{array}{c}\text { Estudiante de la carrera de Trabajo Social. Proveniente de la } \\
\text { comuna de Limache, V Región, Chile. Estudió en un Colegio } \\
\text { Particular Subvencionado. }\end{array}$ \\
\hline
\end{tabular}


Estos estudiantes fueron considerados como informantes claves para el estudio, detallándose los siguientes atributos:

- estudiantes de la cohorte 2015 actualmente matriculados de la universidad, que están cursando su cuarto semestre académico;

- voluntarios que respondieron a la convocatoria de participación para las entrevistas individuales.

Se utilizaron diversas técnicas de recogida de la información; como entrevistas en profundidad, diario de campo y revisión documental. Con el fin de identificar los principales instrumentos de recogimiento de informaciones con los entrevistados se le ha asignado códigos descriptivos a su función.

\section{RESULTADOS DE LA INVESTIGACIÓN}

Emergió una primera meta-categoría que evaluó a la universidad en sus aspectos más generales. Esta primera meta-categoría se denominó Institución, y es explicada por dos categorías que reconstruirán las vivencias que tuvieron estos estudiantes en su primer año universitario. Estas son: opinión sobre la universidad, infraestructura.

\section{OPINIÓN SOBRE LA UNIVERSIDAD}

En general, los estudiantes expresaron tener una satisfacción general con los servicios prestados y la formación recibida por la universidad. En este aspecto, los estudiantes señalaron que rescataban la disposición de algunos docentes para entregar su ayuda en momentos claves, para orientarlos con respecto a las dudas académicas o simplemente para reforzar algunas enseñanzas. La amabilidad y la confianza inspirada por los docentes ayudaron a cumplir las expectativas y necesidades de los estudiantes, incrementando el nivel de satisfacción global con el personal de la universidad; los estudiantes en su proceso de integración a la vida universitaria; "a mí me encanta la universidad, me gusta, la encuentro bien, es práctica, igual la encuentro como cercana, no tan burocrática como yo pensaba que iba a ser" (Estudiante 6).

\section{Infraestructura}

Aunque se han mencionado aspectos positivos de la Institución que se han percibidos como influyentes, los datos revelan que los estudiantes experimentaron sentimientos negativos de rechazo y de crítica frente a las condiciones materiales que presentan las infraestructuras de la universidad. En consecuencia, los estudiantes fueron enfáticos al expresar una crítica material y comunicacional respecto a la segmentación de la institución a lo largo de la ciudad, lo que ha generado distanciamiento, físico, emocional, educativo y cultural; "quizás tal vez me aborrece que estemos tan segmentados a lo largo de la ciudad, eso no me gusta de la universidad, y cada vez más nos separan aún más, cada vez compran más instituciones lejos, se trasladan carreras y eso no me gusta" (Estudiante 3). 
Por otro lado, está la preocupación de los estudiantes por sentirse ajenos a otros espacios físicos de la universidad, declarando que la universidad material y educativamente se compone de pequeñas instituciones que velan por sí mismas (cada escuela, cada edificio apuntan a la particularidad de su realidad).

Finalmente, los estudiantes a pesar de tener una visión crítica frente a la condición material de la infraestructura de la universidad, perciben un sentimiento de cercanía con el tipo de convivencia que han establecido en las infraestructuras de sus carreras o sedes. En ellas, han cultivado un cierto nivel de empoderamiento con el ambiente familiar que se desarrolla en las micro-comunidades, siendo una muestra beneficiosa para generar un fuerte apego y sentido al compromiso con su comunidad. Claramente estas vivencias son opiniones subjetivas y personales, pero demuestra que existe cohesión ante el sentido familiar que han desarrollado en sus ambientes educativos.

\section{COMUNIDAD UNIVERSITARIA}

La segunda meta-categoría se denominó Comunidad Universitaria, esta se dividió en cuatro categorías: convivencia, participación y compromiso, agrupación heterogénea, pertenencia e identidad.

\section{Convivencia}

Los estudiantes reconocieron la capacidad de interacción de sus comunidades locales (micro comunidad) para convivir dentro de los marcos de respeto, reconocimiento y de valorar y aceptar las diferencias. Las vivencias de este tipo de convivencia fueron percibidas como beneficiosas, mostrando que se genera en ellos un fuerte sentido de aceptación y comodidad para proyectar sus expectativas personales y profesionales, así como para establecer relaciones emocionales; "lo que me di cuenta es que todos tenemos algo en común, a todos nos gusta casi las mismas cosas, tenemos los mismos gustos, pensamos igual, no sé si será porque estamos dentro de la misma carrera" (Estudiante 7).

Los elementos primordiales que vinculan buena convivencia son la familiaridad con la que los estudiantes se vinculan con sus pares, generando un ambiente grato y la sensibilidad con que se asocian a otros actores de la comunidad permitiendo estimular el desarrollo de sus habilidades profesionales; "ya como que creamos un grupo de amigos, igual nos ayudamos siempre [...] al final estoy como más tranquila este segundo año, como que me siento mejor que el primero" (Estudiante 3).

La existencia de una convivencia positiva en sus micro-comunidades es lo que favorece un clima agradable para la apropiación de conocimientos, actitudes y destrezas que necesitan para tener un óptimo nivel de desempeño. Este escenario positivo debiese facilitar los procesos de enseñanza-aprendizaje, las buenas interacciones con todos los otros miembros de la comunidad, la positiva recepción a nuevos miembros externos a la comunidad, permitiendo una comunicación interpersonal y social con ellos; "Cuando llega alguien de intercambio se sorprende por lo mismo porque todos conversamos con todos y todos nos conocemos" (Estudiante 9). 
También se reconocen algunas falencias que presenta la universidad para fomentar transversalmente la convivencia de toda la comunidad educativa. Para algunos entrevistados la universidad presenta una comunidad fragmentada, asociada a diversos espacios físicos alejados unos de otros, que impiden comunicarse constantemente, produciendo un distanciamiento afectivo, educativo, social entre los estudiantes de la misma casa de estudios. Este fenómeno para los estudiantes no fue ajeno a su proceso de inserción a la vida universitaria, convirtiéndose en una sorpresa y en una preocupación constante, ya que ellos poseían otras expectativas sobre la convivencia universitaria; "la universidad como que está tan separada, una facultad en Reñaca, otra facultad en el Hucke, otra facultad en Playa Ancha, es decir una universidad muy fragmentada" (Estudiante 3).

Las dificultades de integración entre las micro-comunidades tienen relación directa con:

- la inexistencia de un espacio físico central, como puede ser una sede central o casa central, que agrupe a distintas carreras;

- la presencia de diversas sedes-islas distribuidas a lo largo de dos comunas.

Ambas dificultades han limitado las normas de convivencia de las distintas comunidades por medio del aislamiento comunicacional y educativo, generando un ambiente de lo ajeno entre los estudiantes que visitan otras sedes; "al final al estar todas las carreras en la suya y no hay instancias de cómo hacer una universidad o tener en este caso una casa central que digamos [...] te sientes ajeno, me siento un visitante" (Estudiante 5).

Finalmente, los estudiantes mantienen la expectativa de que la Universidad genere un compromiso a mediano plazo para formar instancias transversales de reunión entre toda la comunidad.

\section{Participación y compromiso}

En términos generales existe una alta satisfacción por la participación universitaria, donde conviven y se respetan las identidades propias de cada sujeto y existe una alta tolerancia a la diversidad de visiones del mundo. Esta posibilidad de involucrarse y participar en espacios locales permite que los estudiantes puedan valorar la calidad de convivencia que tiene la comunidad para compartir ideas, proyectos y trabajo colectivo, y que las distintas percepciones de un fenómeno puedan ser escuchadas con respeto y consideración; "por lo menos mi opinión de Trabajo Social es así, por los programas yo creo que, siempre te mandan correos "participa, participa aquí, participa allă", o siempre dicen "hay invitaciones, hay seminarios; ven participa con nosotros"; yo creo que cierta forma ellos fomentan la comunidad" (Estudiante 6).

Aun así cabe señalar que la conformación de una comunidad participativa está estructurada en diversas micro-comunidades aisladas físicamente unas de otras (infraestructura fragmentada por distintas carreras y/o facultades), donde el lazo social, cultural y académico posee dinámicas diferentes. Esto, para los estudiantes, posee distintas perspectivas sobre la participación dentro de la universidad, estructurando esa heterogeneidad que simboliza la preocupación del beneficio de lo individual 
(carrera) por sobre lo colectivo (universidad en su conjunto), percibiendo así que las problemáticas de lo local pasen a ser una prioridad; "igual estar así tiene sus pros y sus contras. [...] dentro de sus pros es que es como súper familiar. Y también uno espera como conocer gente [...], yo creo que la Universidad debería encargarse de crear instancias donde las carreras participen" (Estudiante 2).

De este modo, existe un interés estudiantil de fortalecer los lazos y vinculación entre las carreras, manifestando la necesidad de construir una red universitaria que supere las realidades micro que presenta cada carrera e infraestructura, y proyectarse hacia un trabajo colaborativo macro y transversal; "que se sientan parte de la universidad, que se conozcan, que compartan las mismas actividades, que se genere esta red de convivencia, que se hagan lazos y se fomente la participación en toda la universidad" (Estudiante 9).

Por otra parte, gran parte de los entrevistados valoraron la entrega de herramientas y apoyo emocional que el programa disponía para su nivelación, por lo que el nivel de participación en estos casos fue constante en el tiempo. Durante su experiencia, ellos establecieron un compromiso personal frente a la necesidad de cumplir sus expectativas académicas, sociales y emocionales, lo que arrojó un alto nivel de satisfacción personal que bordea el sentimiento de tranquilidad ante las adversidades que puedan presentarse a futuro; "Si iba siempre, igual era bueno porque por ejemplo había cosas que los profes no sabían explicar bien. Igual la chica con quien nos tocó nos explicaba súper bien las cosas" (Estudiante 5).

El nivel de compromiso se fue formando a partir del desarrollo de las actividades y el trabajo de los mentores y profesionales del programa, produciendo en los estudiantes un sentimiento de gratitud por el interés demostrado a través del apoyo brindado;

entonces ahí empezamos a saber más del programa después nos empezaron a comunicar, hacer talleres ahí mismo en la escuela, conocimos a los mentores y profesionales y ahí empecé a participar de los talleres que eran de comunicación oral, de cómo manejar los tiempos y esas cosas. (Estudiante 8)

También surgieron otras opiniones que indicaba que algunos estudiantes tuvieron dificultades para participar de las actividades del programa por la gran carga académica o porque se volvió una asignatura más;

Si yo creo que todos los que pertenecimos a la tutoría sentíamos eso; no era como ir a gusto como "hoy día nos toca las mentorías" "vamos a pasarla bien". Era algo más obligatorio que teníamos, y al final de cuentas todo lo que se hace obligatorio y algo extra se vuelve aburrido, se vuelve rutinario y a la gente no le gusta. (Estudiante 3)

La percepción de los estudiantes sobre "lo rutinario" del programa provocó que algunos estudiantes desertaran de las actividades, priorizando sus asignaturas por ende no valorando positivamente los objetivos de apoyo del programa; "Conmigo era nulo el trabajo y le dije bueno yo puedo ir y me topaba con una clase muy 
importante, entonces priorice mis notas antes que el programa, tampoco lo veía como muy importante y formal era como muy informal" (Estudiante 6).

\section{Agrupación heterogénea}

Las relaciones que se generan inicialmente dependen de la compatibilidad de gustos, de intereses, de ideas que presentan los estudiantes. Por lo tanto, el factor de lo común determinará la profundidad de la relación afectiva que establezcan los integrantes del grupo. En el caso de los entrevistados, estos señalaron que inicialmente se agruparon con compañeros con quienes compartían lugares de procedencia o gustos puntuales, pero a medida que los fueron conociendo más, optaron por distanciarse de algunos compañeros para evitar conflictos a futuro.

La reestructuración de grupos o el surgimiento de nuevos grupos es un fenómeno que se da naturalmente durante el transcurso del primer año y responde a filtrar minuciosamente las confianzas y la convivencia afectiva que desarrollan los estudiantes, donde los miembros de ese grupo presenta una identidad colectiva; "ya ese día nos armamos un grupito de las niñas que venían de los sectores más cercano y con algunas me distancié, ya conociendo mejor a las personas como que uno tiende a alejarse un poco por ciertas opiniones o ciertas acciones" (Estudiante 8).

La conexión que presentan los estudiantes en sus grupos aporta en la disposición de generar espacios que permitan la conversación y discusión en torno a materias o en relación a la constitución de grupos de estudios que ayudan a los estudiantes que presentan algún tipo de desventaja académica; "tengo un grupo que nos juntamos a estudiar, que hacemos mapa y esquemas, que nos juntamos en todos lados y nos hacemos preguntas [...] ya no es tan individual y autodidacta el aprendizaje, ahora hay más gente con quien estudiar" (Estudiante 9).

La forma de interacción que adoptan los estudiantes dentro de la vida universitaria ha sido por medio de agrupaciones de carácter heterogéneos que cumplen con finalidades de aportar a la satisfacción de intereses y proyectos comunes. Por lo general, este tipo de agrupamiento nace como una iniciativa implícita durante el proceso de ingreso a la universidad con el fin de poder adaptarse al ambiente universitario.

\section{Pertenencia e identidad}

Entre las valoraciones expresadas por los entrevistados hubo una categoría que constituyó una opinión generalizada y que valoraba el sentido de pertenencia que poseían los estudiantes en sus micro-comunidades. Este sentido de pertenencia significó para los estudiantes conocer y reconocer que aquello que los identificaba con su micro-comunidad establecía los marcos de interacción que tendría con los otros actores de la comunidad de aprendizaje de su carrera y de la universidad.

La internalización de ¿con qué me siento más identificado? generó en los estudiantes compromisos afectivos, educativos y sociales con el simbolismo de ser integrante activo de una comunidad pequeña (micro-comunidad), que representaba autónomamente un sello de convivencia dentro de la institución, por el cual los estudiantes ejercían las prácticas de convivencia universitaria. 
Todos los entrevistados manifestaron que se sentían más representados por sus carreras/escuelas que por la universidad, un sello que perfila la identidad autónoma de cada carrera por sobre el sello de la que la propia universidad quiere imprimir a sus egresados. Por consiguiente, vemos mermada la relación de la identidad del estudiante-institución a cambio de una relación con las micro-comunidades autónomas que suplantan la noción de universidad - en el sentido de universal - y que por tanto genera una relación afectiva y efectiva entre el ambiente de la comunidad con el estudiante; "Sí, es como un sello es que siento que las carreras, que cada carrera tiene un perfil de estudiantes [...] cada uno tiene su perfil, su carrera. Como el perfil de las humanidades, como que somos revolucionarios" (Estudiante 2).

Como consecuencia, la construcción de identidad de comunidad universitaria queda sujeta a la gestión de cada micro-comunidad o comunidades secularizadas que dependiendo de sus condiciones subjetivas (valores, personalidades, comunicación) y objetivas (ubicación, infraestructura, gestión institucional) van conformando la relación afectiva entre los miembros de la comunidad; "Yo soy de Trabajo Social, no de la universidad [...] yo estudio Trabajo Social y soy una estudiante de la carrera y me gusta [...] decir que soy de la universidad como que no le veo la relevancia" (Estudiante 9).

Con ello se entiende que existe una ruptura identitaria entre lo local y el espacio ampliado (universidad), produciendo dos perspectivas de este fenómeno:

- el espacio local suplanta y se apropia de la tarea de construir identidad comunitaria;

- la institución es incapaz de establecer estrategias comunitarias que aporten a la construcción de la identidad universitaria institucional.

\section{EVALUACIÓN DEL PROGRAMA}

La tercera meta-categoría fue la evaluación del programa me quedo en la universidad. El análisis fue dividido en cuatro categorías que creemos son esenciales para explicar vivencias que tuvieron los estudiantes dentro este programa de acompañamiento universitario: Desconocimiento del programa, Autoexclusión del programa, Satisfacción por el programa, Sugerencias de mejora, Apoyo, Mentorías, Propuesta.

\section{Desconocimiento del programa}

Podemos apreciar en esta categoría que existe mucha coincidencia en los discursos de los estudiantes que desconocieron la existencia del programa de acompañamiento psico-social "Me quedo en la universidad" como un elemento importante en su formación inicial, por lo que la invitación a participar dentro del estudio fue tomada con sorpresa; "Y nos entregaron como un sobre, y nos explicaron así con una hoja lo que era el programa, que habíamos sido seleccionados, por ciertas características que a nosotros nunca nos dijeron" (Estudiante 2).

A pesar de haber desconocido su existencia, de no haber tenido claro sus objetivos y su plan de acción, la invitación fue recibida de buena manera por los estudiantes como una opción atractiva. Es más, a medida que los estudiantes fueron participando activamente del programa vivieron experiencias que se tornaron en 
opiniones positivas; "Y la verdad fue como interesante porque era algo nuevo [...] y yo no sabía que iba a tener en la U. Ya cuando fuimos sabiendo que era para que nos quedáramos en la universidad, igual era bueno más si era para recibir ayuda con profesores y todo eso" (Estudiante 3 ).

Pero ello no quita que para otros estudiantes la invitación no generó ninguna expectativa inicial: "Sí, entonces no sabía de qué se trataba en realidad entonces como que no tenía una expectativa más allá, pero ya de que me fui incorporando un poco más me di cuenta de que era para nivelar, para tener un apoyo tanto a la transición del primer año de Universidad” (Estudiante 8).

Queda claro que los estudiantes ingresaron a la universidad sin saber de este apoyo para la retención, esta política educativa por tanto podría ser comunicada en la fase de orientación en los liceos y colegios.

\section{Autoexclusión del programa}

Algunos estudiantes señalaron el escaso de interés que produjo el programa, evidenciando que la beca no fue lo bastante atrayente para algunos estudiantes. De este modo, la forma de interpretar este fenómeno se limita inicialmente a que las estrategias convencionales a las que optó el programa no fueron lo suficientemente didácticas para brindar un sentido distinto a la beca, ya que los estudiantes asociaban naturalmente el programa a un ramo adicional; "Claro, si era como [...] una rutina, un ramo más" (Estudiante 3), "Sí, tuve mentores, pero la asistencia era súper baja, de hecho mi grupo no iba casi nunca" (Estudiante 3), "Igual eran súper pocas las niñas que iban. Incluso iban niñas que no pertenecían al programa y que aprovechaban de estar ahí" (Estudiante 8). Esta visión perjudicó la asistencia de los estudiantes becados al programa de apoyo, originándose una autoexclusión.

\section{Satisfacción por el programa}

En voz de los estudiantes que efectivamente asistieron al programa manifestaron lo beneficioso que fue para su desarrollo integral académico y social, reconociendo de buena manera la labor realizada por los distintos actores que contribuyeron a la realización del programa: compañeros, mentores, académicos, equipo profesional. El programa ayudó a fortalecer la integración académica y al desarrollo de un tejido social dentro de la Universidad, transcendiendo y permeando otros contextos asociados a la IES.

Por tanto, las distintas actividades que realizó el programa de becas han cumplido su función de nivelación, quedando plasmado en las opiniones que vertieron los entrevistados cuando se les preguntó acerca de si los talleres les ayudaron en su rendimiento académico; "Entonces, en lo que es particular a mí, me sirvió bastante ese programa” (Estudiante 8).

El apego estudiante-mentor que generó el programa es destacado como un elemento de gran satisfacción, ya que el trabajo entre pares les resultó un apoyo importante. En este punto conviene destacar a disposición profesional del equipo del programa, quienes brindaron su ayuda cuando el estudiante necesitó informarse y/u orientarse; 
Claro, $[\ldots]$ entre estudiantes, me gustó el tema de la beca porque siento que fue una ayuda al estar en la U, o sea uno entra como individual por así decirlo y recibir una ayuda de parte de un equipo se agradece mucho, el Dany me explicaba, me orientaba y todo eso, igual eso fue como bueno. De verdad agradezco que me hayan dado esa beca. (Estudiante 3)

Como menciona Bensimon (2007), la capacidad de ayuda de un agente educativo está fuertemente influenciada por el conocimiento que tenga del ámbito cultural de los estudiantes y en la medida de que se de este conocimiento, el agente estará capacitado para fortalecer el autoestima y proveerá de recursos necesarios para que el estudiante sea exitoso; "esto de enseñarte de otra forma, ayudaba a que tú no te fueras de la universidad y entendieras y que hicieras que no te rindas, no frustrarte, era como ya lo aprendí puedo seguir; entonces igual creo que fue un gran ayuda; sí, a mí me sirvió" (Estudiante 5).

Los mismos estudiantes han destacado las características de manejo comunicacional y disciplinario que los profesionales y mentores del programa tuvieron con ellos. El hecho de saber escuchar, preguntar y explicar ha fortalecido el clima de confianza para que ellos puedan proyectar un apoyo emocional distinto a la familia, compañeros o cercanos. Este canal de comunicación de trato interpersonal de tipo informal aportó en el proceso de la integración del estudiante con su medio y la necesidad de compartir con sus compañeros;

En cuanto a la planificación de actividades, a las personas que conocía y detrás de ellos lo evalué con la mejor nota posible, para mí fue lo mejor, fueron súper... fueron profesionales pero también fueron amigos, compañeros con los que uno podía tener un acercamiento más profundo. (Estudiante 8)

La construcción por ende de una comunidad de aprendizaje fue lograda en la medida que todos los actores se involucrasen de forma efectiva y afectiva.

\section{Sugerencias de mejora}

Comprendiendo las diversas particularidades que se han hecho presentes en cada una de las coyunturas de los estudiantes, es que se hace hincapié en que el estudiante está preocupado por señalar las deficiencias que ha presentado el programa durante su desarrollo. Con miras a mejorar exitosamente el programa, los estudiantes han comprendido su naturaleza piloto, por lo que es normal que presente errores al momento de implementar estrategias de apoyo e intervención. Por tanto, son conscientes de esta práctica de aprender por medio del ensayo y error; "siento que es algo como que recién está empezando, y nunca va a ser perfecto cuando se está empezando. Pero siento que podrían haber hecho más cosas. Y no tantos talleres como de lo académico" (Estudiante 2).

Los estudiantes buscan sistematizar el acompañamiento continuo para solucionar las deficiencias y problemáticas que se les presente en su desempeño académico, por lo que para ellos resulta contradictorio tener un programa de apoyo y acompañamiento que disponga de una metodología de trabajo que sea inconexa 
a sus necesidades cuando realmente necesitan un trabajo permanente y continuo. Una idea que se hace latente es elaborar un plan de trabajo académico que les permita avanzar progresivamente en su desarrollo de habilidades académicas, sociales y profesionales; "la metodología esta mejorable [...] entonces esas ayudantías, esas charlas que se yo esas mismas entrevistas pero ya de una manera de que conozcan ya a la persona y no una vez al semestre" (Estudiante 9).

Otro elemento a recalcar es la falencia que presentó a nivel organizativo del programa al momento de coordinar y gestionar las actividades de corte extracurriculares. Así, los estudiantes manifestaron una desorganización de la beca como una intervención tardía y/o inconexa, no logrando articular debidamente un servicio de orientación que pretendiese continuamente responder a sus necesidades. Ante esto, los mismos entrevistados proponen que la institución debe, en primer lugar, identificar las expectativas de sus estudiantes y trabajar en su cumplimiento; "el año pasado fue algo desorganizado, fue un poco más tardío de lo que pudo haber sido y no todos tenían como esa noción de que tenían un mentor" (Estudiante 1).

Ante estas recomendaciones, Swail (2004) sugiere que cada Institución debe diseñar su propia estrategia de retención de acuerdo con las características particulares que presente la Institución y la comunidad universitaria, por lo tanto sus enfoques teóricos y modelos de análisis debiesen apuntar a esos objetivos. Por tanto, el diseño que presenta este programa debiese tener en cuenta este tipo de consideraciones que los estudiantes de su primera generación señalan para poder mejorar el programa y así dar especial importancia a una Institución que desarrolla planes de acción preventivos y no solamente planes asistencialista de naturaleza correctiva.

\section{CONCLUSIONES Y REFLEXIONES}

Dentro del proceso analítico, se expresó la intención proactiva de proponer algunas modificaciones al programa, específicamente al rendimiento que tiene el quehacer de las estrategias metodológicas que utilizaban para el proceso de acompañamiento continuo. Según Boylan y Saxon (2009), estas prácticas son consistentes con estudios respecto de programas de acompañamiento, los que han demostrado que los cursos intensivos, las tutorías y el asesoramiento individual resultan altamente efectivos para favorecer el rendimiento académico y la permanencia en la educación superior.

En torno a esta concepción, para los entrevistados esta realidad mencionada no fue del todo exacto, ni tampoco similar, porque el contexto que entrelazaba sus necesidades con lo que proponía el programa no concordaban del todo, problematizando el proceso de atención psicoeducativa y el monitoreo permanente.

A consecuencia de ello, la implementación de estrategias de acompañamiento no alcanzó a satisfacer las necesidades de todos los estudiantes, ni tampoco fue un apoyo inmediato, por lo que los estudiantes sugirieron que los cambios próximos del programa debiesen apuntar a contextualizar, entrelazar ampliamente y enriquecer a la partir de las propias necesidades de los estudiantes de la carrera y de la universidad, apostando a un programa que no excluya sino que complemente a todos los estudiantes de la institución. 
Una de las ideas que se destacan de esta propuesta es establecer un acompañamiento continuo y que no sean intervenciones psicoeducativas desfasadas o sin sentido progresivo, ya que el potencial de los profesionales puede ser aprovechado de mejor manera para el monitoreo del plan de trabajo que está relacionado al rendimiento académico de cada estudiante.

Junto con ello la figura del mentor surge como un actor importante y bien valorado ya que permitió conocer y compartir con compañeros de generaciones más antiguas, estimulando su vinculación e integración a la comunidad universitaria. La figura del mentor permitió a los estudiantes de recién ingreso conocer de qué se trataba la carrera y desarrollando estrategias que favoreciesen el éxito académico. A saber, el mentor tuvo un rol de ente consultivo de dudas puntuales sobre la carrera o habilidades académicas y por sobre todo surge como un buen acompañante a modo de hermano mayor en un periodo de transición educativa difícil y compleja.

Comprendiendo que el proceso de incorporación o inserción al mundo universitario supone para el estudiante enfrentarse a diversos desafíos, es que las estrategias de acompañamiento según los entrevistados debiesen apuntar hacia un monitoreo permanente y continuo con los profesionales que acogieran sus necesidades académicas y afectivas, y además plantear un plan de acción que ofrezca soportes de apoyos específicos.

En suma, la sistematización del acompañamiento continuo se puede constituir como una importante herramienta de apoyo para la integración a la vida académica y la orientación de estrategias de aprendizaje para el proceso formativo de los estudiantes de la universidad, y esto los entrevistados lo expresan por medio de sus propuestas de mejoramiento hacia la beca de nivelación académica.

Es interesante destacar que a partir de este estudio focalizado de un programa de atención de apoyo psico-social a estudiantes de primer año de una universidad pública surgen nuevos focos críticos en que las IES deben desarrollar estrategias de mejora:

- uno de ellos se refiere a las políticas públicas que se establecen para el éxito académico, medido por la retención estudiantil y otros factores como la titulación oportuna y el desarrollo de nuevas capacidades que demanda el mercado hacia la formación terciaria y que no siempre es capaz de cumplir de manera satisfactoria;

- otro punto crítico es considerar el impacto que tiene la deserción universitaria al interior de las familias en el plano económico y social, como también el impacto que genera para el propio sujeto que no termina su educación superior;

- la identidad de una institución se juega y se fomenta en la interacción cotidiana, y en el caso de la universidad, sus espacios visibles e invisibles pueden fragmentar o unir a una comunidad universitaria. El trabajo de convivencia e integración debe ser una prioridad en la política y gestión universitaria. Para garantizar la identidad y la participación de los estudiantes debiese de existir un acceso equitativo a todos los espacios institucionales, permitiendo la participación de la comunidad ante la utilización de la infraestructura (aulas, patios, biblioteca, etc.), es decir, las mismas oportunidades para todos y todas; 
- finalmente se hace necesario reflexionar sobre el futuro papel que tendrán las universidades chilenas con la promulgación de la Ley de Educación Superior y la creación de una Superintendencia que fiscalizará sus actuaciones. Con ello se abre la oportunidad histórica de transitar hacia una gestión más trasparente y con mayor participación de todos sus estamentos. La universidad debe ser la suma de buenas prácticas, de trabajo serio y comprometido y por sobre todo de un actuar en humanidad que ilumine las actuaciones de nuestras sociedades.

La importancia del fenómeno de la retención debiese apuntar a un objetivo final, instalar la capacidad del estudiante por lograr su autoeficacia - persistencia - y avanzar para que las condiciones que brindan las IES logré su objetivo, que cada vez más estudiantes cumplan con sus carreras de manera oportuna e ingresen al mundo laboral a mejorarlo.

\section{REFERENCIAS}

AHUMADA, J. En vez de la miseria. Santiago: Editorial del Pacífico, 1958.

ATTINASI, L. C. Getting in: Mexican American Student's perceptions of their college-going behavior with implications for their freshman year persistence in the University. In: ASHE, 1986. Annual Meeting Paper [...]. San Antonio: EE.UU, 1986. (ERIC n. 268 869).

BARRIOS, A. Deserción y financiamiento en las universidades chilenas. 158f. 2011. Tesis (Magíster en Ciencias de la Ingeniería) - Pontificia Universidad Católica de Chile, Santiago, 2011.

BASSO, P. Educación Superior en Chile: El fracaso del modelo neoliberal. Revista Interdisciplinaria de Filosofía y Psicología, Arica, v. 11, n. 37, p. 21-48, 2016. Disponible en: https://dialnet.unirioja.es/servlet/articulo?codigo=6621679. Acceso en: 9 ene. 2017.

BENSIMON, E. The Underestimated significance of practitioner knowledge in the scholarship on student success. The Review of Higher Education, Baltimore, v. 30, n. 4, p. 441-469, 2007. http://dx.doi.org/10.1353/rhe.2007.0032

BOYLAN, H.; SAXON, P. Creating quality developmental education. A guide to the top ten actions community college administrators can take to improve developmental education. 2009. Disponible en: https://docplayer.net/11146286-Creating-qualitydevelopmental-education.html. Acceso en: 25 ene. 2017.

BRAXTON, J. M.; MILEM, J.; SULLIVAN, A. The Influence of Active Learning on the College Student Departure Process: Toward a Revision of Tinto's Theory. The Journal of Higher Education, Ohio, v. 71, n. 5, p. 569-590, 2000. http://dx.doi. org/10.2307/2649260

CAMPBELL ESQUIVEL, J. C. La educación superior en Chile: experiencias de la década de los 80 y sus proyecciones. In: ESQUIVEL, J. (org.). La Universidad, hoy y mañana: perspectivas latinoamericanas. México: ANUIS/UNAM, 1995. p. 113-130. 
CASTILLO, J.; CABEZAS, G. Caracterización de jóvenes primera generación en educación superior. Nuevas trayectorias hacia la equidad educativa. Revista Calidad en la Educación, Santiago, n. 32,p.44-76, jul.2010. https://doi.org/10.31619/caledu.n32.151 CHILE. Ministerio de Educación. Algunos Antecedentes para el Planeamiento Integral de la Educación Chilena. Santiago: Editorial Universitaria, 1964.

CHILE. Ministerio de Desarrollo Social. Estadísticas Educación Encuesta CASEN: Cobertura de educación superior por quintil de ingreso autónomo nacional según sexo. Santiago: Observatorio Social, Ministerio de Desarrollo Social, 2013a. Disponible en: http://observatorio.ministeriodesarrollosocial.gob.cl/casen/casen-documentos. php?c=87\&amp;a=2011. Acceso en: 16 dic. 2016.

CHILE. Ministerio de Educación. Serie Evidencias: Medición de la deserción escolar en Chile. Santiago: Centro de Estudios MINEDUC, 2013b. Año 2, n. 15. Disponible en: https://centroestudios.mineduc.cl/wp-content/uploads/sites/100/2017/06/A2N15_ Desercion_escolar.pdf. Acceso en: 14 mar. 2015.

COnSEjo nACIONAL DE EDUCACIÓn (CNED). Matrícula Sistema de Educación Superior. Chile: CNED, 2018. Disponible en: https://www.cned.cl/indices/ matricula-sistema-de-educacion-superior. Acceso en: 22 abr. 2018.

CUCHACOVICH, I. Retención y movilidad universitaria: Evaluación de impacto de una política. 38 f. 2011. Tesis (Magister en Economía) - Pontificia Universidad Católica de Chile, Santiago, 2011.

DONOSO, S.; DONOSO, G.; FRITES, C. La experiencia chilena de retención de estudiantes en la universidad. Ciencia y Cultura, La Paz, n. 30, p. 141-171, 2013. Disponible en: http://www.scielo.org.bo/pdf/rcc/n30/a07.pdf. Acceso en: 22 mar. 2017. DONOSO, S.; SCHIEFELBEIN, E. Análisis de los modelos explicativos de retención de estudiantes en la universidad: una visión desde la desigualdad social. Estudios Pedagógicos, Valdivia, v. 33, n. 1, p. 7-27, 2007. Disponible en: http://www.redalyc. org/articulo.oa?id=173514133001. Acceso en: 11 ago. 2015.

DURKHEIM, E. El Suicidio: un estudio de sociología. 2. ed. Madrid: Ediciones AKAL, 2012.

ETHINGTON, C. A psychological model of student persistence. Research in Higher Education, v. 31, n. 3, p. 266-269, 1990. Disponible en: http://rhartshorne.com/fall2012/eme6507-rh/cdisturco/eme6507-eportfolio/documents/ethington.pdf. Acceso en: 22 ago. 2015.

GAJARDO, M. América Latina: políticas educativas de acción afirmativa. Situación, tendencias y perspectivas. In: GARCÍA HUIDOBRO, J. E. (org.). Políticas Educativas y Equidad. Santiago: Ford Foundation, UNESCO, UNICEF, Universidad Alberto Hurtado, 2005. p. 101-118.

GOBIERNO DE CHILE. Encuesta Casen 2011: Módulo Educación. Chile, 2011. Disponible en: http://observatorio.ministeriodesarrollosocial.gob.cl/layout/doc/ casen/2013_04_01_Educacion_CASEN_2011.pdf. Acceso en: 16 mar. 2015.

GÓMEZ, J. et al.Metodología Comunicativa Crítica. Barcelona: El Roure Editorial, 2006. 
GONZÁLEZ, L. E. Estudio sobre la repitencia y deserción en la educación superior chilena. Santiago: IESALC-UNESCO, 2005. Disponible en: http://unesdoc.unesco. org/images/0014/001400/140087s.pdf. Acceso en: 14 abr. 2015.

HAMUY, E. El problema educacional del pueblo de Chile. Santiago: Editorial del Pacífico, 1961.

HIMMEL, E. Modelo de análisis para la deserción estudiantil en la educación superior. Calidad de la Educación, Santiago, n. 17, p. 91-108, 2002. http://dx.doi.org/10.31619/ caledu.n17.409

NAVARRETE, S.; CANDIA, R.; PUCHI, R. Factores asociados a la deserción/ retención de los estudiantes mapuche de la Universidad de la Frontera e incidencia de los programas de apoyo académico. Calidad en la Educación, Santiago, n. 39, p. 43-80, 2013. Disponible en: https://scielo.conicyt.cl/scielo.php?script=sci arttext\&pid=S0718-45652013000200003. Acceso en: 8 feb. 2015. http://dx.doi. org/10.4067/S0718-45652013000200003

ORELLANA, V.; SANHUEZA, J.; GAJARDO, F. El futuro es la educación pública: Diagnóstico y propuestas para la construcción y expansión de una red de Educación Superior Pública sin recursos adicionales. Santiago: Fundación Nodo XXI, 2016. Disponible en: https://es.scribd.com/document/329768293/InformeEducacion-Superior-Publica-Nodo-XXI\#from_embed. Acceso en: 8 feb. 2015.

ORGANIZACIÓN PARA LA COOPERACIÓN Y EL DESARROLLO ECONÓMICO (OCDE). La educación superior en Chile. París: OCDE, 2009. Disponible en: http://www7.uc.cl/webpuc/piloto/pdf/informe_OECD.pdf. Acceso en: 22 mar. 2017.

PASCARELLA, E. College environmental influences on learning and development: A critical review and synthesis. In: SMART, J. C. (org.). Higher education: Handbook of theory and research. Nueva York: Agathon, 1985.v. 1. p. 127-153.

RIESCO, M. ¿Capitalista o Rentistas? El Mostrador, Santiago, 2010. Disponible en: http://www.elmostrador.cl/noticias/opinion/2010/09/10/\%C2\%BFcapitalistas-orentistas/. Acceso en: 16 mar. 2016.

SÁNCHEZ, M. Reforma Educacional y deserción escolar. El Mostrador, Santiago, 2015. Disponible en: http://www.elmostrador.cl/noticias/opinion/2015/02/08/reformaeducacional-y-desercion-escolar/. Acceso en: 9 feb. 2015.

SCHIEFELBEIN, E. Diagnóstico del sistema educacional chileno en 1970. Santiago: Universidad de Chile, Departamento de Economía, 1976.

SERVICIO DE INFORMACIÓN DE EDUCACIÓN SUPERIOR (SIES). Listado de instituciones de educación superior vigentes. Santiago: Servicio de Información de Educación Superior,2013. Disponible en: http://www.mifuturo.cl/images/Instituciones/ instituciones_vigentes_agosto_2013.xls. Acceso en: 13 mar. 2015.

SPADY, W. G. Dropouts from higher education: An interdisciplinary review and synthesis. Interchange, v. 1, n. 1, p. 64-85, 1970. https://doi.org/10.1007/ BF02214313 
ST. JOHN, E. P. et al. Economic Influences on Persistence Reconsidered: How can finance research inform the reconceptualization of persistence models? 2000. Disponible en: http://citeseerx.ist.psu.edu/viewdoc/ download?doi=10.1.1.717.6133\&rep=rep1\&type=pdf. Acceso en: 12 dic. 2015.

STAKE, R. Investigación con estudio de casos. Traducción: R. Filella. Madrid: Morata, 1998.

STRATTON, L.; O'TOOLE, D.; WETZEL, J. A multinomial logit model of college stopout and dropout behavior. Economics of Education Review, v. 27, n. 3, p. 319331, 2008. https://doi.org/10.1016/j.econedurev.2007.04.003

SWAIL, W. The Art of Student Retention. A Handbook for Practitioners and Administrators. In: ANNUAL RECRUITMENT AND RETENTION CONFERENCE, 20., 2004. Texas: Educational Policy Institute, Texas Higher Education Coordinating Board, 2004. Disponible en: https://files.eric.ed.gov/fulltext/ ED485498.pdf. Acceso en: 18 dic. 2015.

TILLMAN, C. Barriers to student persistence in higher education. Didache: Faithful Teaching, v. 2, n. 1, 2002. Disponible en: https://www.whdl.org/sites/default/files/ v2n1_Tillman.pdf. Acceso en: 10 ene. 2016.

TINTO, V. Dropout From Higher Education: A Theoretical Synthesis of Recent Research. Review of Educational Research, v. 45, n. 1, p. 89-125, 1975. Disponible en: http://journals.sagepub.com/doi/10.3102/00346543045001089. Acceso en: 14 ene. 2016. https://doi.org/10.3102\%2F00346543045001089

\section{SOBRE LOS AUTORES}

Pablo Castillo Armijo es doctor en educación y sociedad por la Universitat de Barcelona (España). Professor de la Universidad de Talca (Chile). E-mail: pablo.castillo.armijo@gmail.com

Tomas Morales Zárate es licenciando en educación por la Universidad de Valparaíso (Chile).

E-mail: tomasmoralesz@gmail.com

Carlos Miranda Carvajal es mestre en evaluación educacional por la Universidad de Playa Ancha (Chile). Profesor de la misma institución. E-mail: cmc.mira@gmail.com

Recibido el 8 de julio de 2018 Aprobado el 19 de julio de 2019 